\title{
DNA perspectives of fixed and paraffin embedded human tissues as resource materials for the identification
}

\author{
Sami Ullah ${ }^{1}$, Rakesh K. Garg ${ }^{1^{*}}$ and Farida Noor ${ }^{2}$
}

\begin{abstract}
In addition to the diagnostic and therapeutic use of excised human tissues in fixatives, the fixed tissues represent an invaluable resource that provide an incredible opportunity for the assessment of genetic studies in situations where subjects are no longer alive. Purposely the fixation serves to prevent the cellular changes as well as a resistance to cellular alterations that occur due to abrupt ligation of circulation during excision. Importantly DNA extracted from these tissues can be used in the identification of a person such as in cases of paternity testing, abduction or soldier missing in action, in crime cases where biological evidence is available for comparison, identifying mismatched specimens for re-evaluation and more outstandingly in insurance cases. However, the DNA recovery from these samples along with its amplification is a challenging task due to various factors thereby limiting the use of these preserved specimens for forensic DNA related investigations. In order to clarify various doubtful reproaches in different cases where justice had to be done from the challenging samples of fixed specimens, the present paper aimed not only on the issues concerning with these invaluable specimens, but also on varied interesting nature of case reports so that better discrimination is achieved in the areas of concern.
\end{abstract}

Keywords: Forensic, DNA analysis, Paraffin-embedded tissues, Formalin fixed samples, Fixation

\section{Background}

According to the expertise working on fixed human tissue specimens, roughly a billion or more tissue samples stored in hospitals and tissue banks around the world, comprise formalin-fixed (FF) and formalin fixed paraffin-embedded tissues (FFPET) (Blow 2007) purposely excised out for histopathological examination of disease progression details; or are taken during autopsies performed to establish the cause of death. The role of this fundamental process (fixation) thus constitutes an important element in medical diagnostic procedures and clinical research primarily conceived to facilitate microscopic analysis to study the diseases like cancer. Alongside this, the purpose also remains to prevent the autolysis, putrefaction and degradation of the tissue and tissue components as well as to preserve it in a hardened lifelike state (Farrugia et al. 2010). However, as the never ending advancement in

\footnotetext{
* Correspondence: rkgvpbi11@yahoo.com

'Department of Forensic Science, Punjabi University, Patiala, Punjab 147002, India

Full list of author information is available at the end of the article
}

molecular biology techniques progressed, a challenged need was felt to assess these specimens for forensic DNA related investigations. Needful to say, that the identification using these fixed specimens as a source for DNA typing has got an ever increasing importance in the forensic science. The reason being DNA extracted from these fixed tissues finds its greatest applications in retrospective studies notably in sudden unexplained death where FF and FFPET is a unique source of genetic material and therefore represents an invaluable resource for genetic studies in situations where subjects are no longer alive as reported in the literature (Chugh et al. 2004; Doolan et al. 2008). DNA extracted from these tissues finds great applications in the identification of a person such as in paternity testing, abduction or soldier missing in action, in crime cases where biological evidence is available for comparison, identifying mismatched specimens for reevaluation (Budimlija et al. 2009; Raina et al. 2011) or to clear doubtful approaches regarding contamination issues (Popiolek et al. 2003) and in insurance cases (Liu et al. 2009). However because of the declining state of 
these samples and a lack of standardized preservation method, DNA recovery from these samples along with its amplification, is a challenging task due to the apparent nucleic acid degradation by way of aging of the specimen in the fixative or the $\mathrm{pH}$ of the fixative by gradual changes of formaldehyde to formic acid thereby hydrolyzing the DNA and extensive modification that affects both the quality and quantity of DNA (Gilbert et al. 2007; Gillio-Tos et al. 2007; Huijsmans et al. 2010). It has also been shown that formation of methylene bridges between the amino groups of purine and pyrimidine bases as well as between these nucleotide bases and histones is induced by formalin. Furthermore, scission of the phosphodiester backbone of DNA may also occur, resulting in recovery of fragmented low molecular weight DNA (Romero et al. 1997).

As long as high molecular weight DNA is not required like in RFLP approaches, DNA extracted from formalin fixed and paraffin embedded tissues can be made suitable for polymerase chain reaction (PCR) analysis significantly by virtue of its sensitivity of detection (by PCR usage) and an ability to analyze minute samples, provided standardized extraction protocols are strictly followed. However, the success of PCR amplification of genomic DNA from FF and FFPETs may be critically dependent on a variety of factors: including handling of specimens before tissue fixation, type of fixative, fixation time, storage temperature and storage time. Though there is widespread DNA typing acceptance in forensic science field importantly due to its sensitivity of detection (by PCR usage) and an ability to analyze minute samples. Fixed specimens a potential resource for DNA extraction cannot be used as a source of DNA for RFLP approaches where HMW DNA is a pre requisite for successful typing results particularly because of degradation that eventually lead to fragmentation.

Fragmentation of DNA that is probably caused by virtue of low $\mathrm{pH}$ and DNA protein cross linkages mostly renders it to a poor performer in downstream applications. Like DNA quantity, degree of fragmentation also varies and is mostly a function of sample preservation method. The extraction of DNA from these degraded sources (fixed specimens) is therefore a challenging research aspect in forensic field. As a matter of fact, when there are only a few template molecules available for PCR, stochastic amplification would occur where the degree of its effect would indirectly be related to the number of template molecules. For the alleles of short tandem repeat (STR) loci that are based on a varied number of tandem repeats, stochastic effects manifest as a substantial imbalance of two alleles at a given heterozygous locus, allelic dropout, and increased stutter. Many strategies are available that address the above mentioned phenomenon to generate a successful DNA profile which are however not considered in this paper and needs to be studied separately.

\section{Main text}

Apart from original purpose of fixed and embedded human tissues for disease progression study, these specimens represent a resource for the identification particularly in cases which demand special considerations. Provided an opportunity for analysis is not lost with an impossibility assessment, the forensic DNA examination of these samples might well help in clarifying doubtful reproaches particularly in cases where these specimens represent last sources for the identification. Due emphasis was laid on the interesting nature of below mentioned case reports so that the findings may accurately safeguard the issues (Banaschak et al. 2000; Islam et al. 2013; Reshef et al. 2011; Robino et al. 2006):

1. A suspicion regarding block exchange, labeling error, or a technical error was raised in a case where in histopathological reports of a patient didn't reveal any tumor residue in the post-surgical tissue block, although all the pre-surgery clinical evidence had diagnosed a high grade breast carcinoma. Hence in order to explain the cause for the negative results so obtained, multiplex short tandem repeat analysis of paraffin block the only evidence of the patient in the laboratory and the patient's fresh blood was undertaken. However multiplex AmpFlSTR analysis capable of amplifying 15 STR loci and amelogenin (for sex determination) was able to amplify only 9 and 8 loci, respectively from two paraffin processed blocks, and the amplification of all the 15 loci in case of blood. The absence of tumor residue in the post-surgical tissue block was therefore attributed/ identified to be a technical retrospective error in the gross examination of the post-surgical tissue as a wrong part (normal) was selected for the histopathological analysis. The DNA fingerprinting thus helped in justifying the therapy given to the patient along with no evidence of sample swapping or a labeling error.

In this case the random matching probability of the sample where all loci were amplified was calculated to be 1 in 5.19E15, 8.83E13, and 1.52E14 in Caucasian population, Asian population and East Indian populations, respectively. The values were sufficiently high to distinguish the individuals. In case of sample where 9 loci, were amplified the values were 1 in 1.57E6, 4.43E4, and 1.36E5 for Caucasian population, Asian population, and East Indian populations, respectively. Similarly $2.56 \mathrm{E} 6$, $1.89 \mathrm{E} 6$, and $1.27 \mathrm{E} 6$ were the random matching probability values for the sample where 8 loci were amplified for Caucasian population, Asian population, and East Indian population, respectively. Due to remarkable DNA damage in both the 
paraffin blocks partial DNA profiles were obtained. However a critical evaluation of 8-9 loci was sufficient for the conformational results (Raina et al. 2011).

2. A histological section of a patient with no clinical evidence of malignancy showed a tiny fragment of high-grade carcinoma that made the patient doubtful whether it was truly what has been shown in the report or simply an issue of contamination. To clear the suspicion and to trace the contaminant to its source where its presence was felt in 3 consecutive histologic sections and in the paraffin block of the patient, comparative STR analysis to paraffin-extracted tissue samples obtained from the polyp, the suspected contaminant, the patient's additional cervical biopsy specimen, and the putative source of contamination was performed. The results without a reasonable doubt confirmed contamination with the detection of its origin in a tumor processed earlier.

Due to the extremely small and compromised complex nature of tissue by fixation and embedding, allelic dropout (false homozygosity) was seen in the amplification of few target loci probably by virtue of stochastic fluctuations of the target DNA. However, statistical interpretation of the amplified loci was sufficient to attach a match with a probability of 1 in approximately 13.8 trillion blacks, 1.06 trillion Caucasians, 788 billion Hispanics, and 655 million Asians, thereby tracing the origin of the oncogenic fragment to only one same person leaving the patient free from additional treatment burden (Popiolek et al. 2003).

3. A businessman died due to stomach cancer and was subsequently cremated. To be an heir at law, so that legacy and continuity in business could be claimed, the dead man's son was asked to prove his parenthood because of discrepancy of name in his overseas ID card and mainland ID card. Accordingly the son submitted the gastric cancer tissues (only remains) of his father that were surgically removed and embedded in a paraffin block as source of DNA for paternity testing. After partitioning cancerous tissue from normal adjacent tissue DNA was extracted with QIAamp DNA Blood Mini Kit, the 16 STR loci was amplified by two commercial kits of Sinofiler ${ }^{\bullet}$ (ABI) and Powerplex 16 (Promega), respectively. When STRs profile of normal tissue was compared with the STR of son's blood sample, a final conclusion was drawn that the donor of the paraffin embedded tissues is the biological father of the son with a paternity index of 206,438.36 and hence decisive results were obtained from fixed tissues which otherwise would not have been possible (Liu et al. 2009).
4. In cases where interchanging of tissue samples is suspected, the consequences are always followed by a dramatic surprise of a patient. Due to false negative finding results a patient leaves out necessary therapy; whereas false positive findings may subject him to suffer unnecessary operations and chemotherapies. A treating gynecologist along with a pathologist was suspected of incorrect diagnoses and interchanging of tissue samples in breast cancer related cases. To clarify the reproaches the attorney's bureau confiscated histological slides, paraffin blocks and formalin fixed tissues from a privately owned pathological laboratory. Accordingly the confiscated materials were subjected to DNA examinations and compared to reference material of the patients for the proof of interchanging tissue samples. For diagnoses appearing to be surprising for a certain age or for preexisting diseases it becomes important to take into account the possibility of interchanging of tissue samples where its consequences are always dramatic for the patient as said earlier (Banaschak et al. 2000).

5. It is seen that on many occasions diagnosis and treatment are delayed apparently when multiple biopsies taken from the same patient unfortunately give conflicting results at multiple centers. In two cases of this nature molecular genetic testing of fixed bioptic material was used for mitigating the issue so that specimen identity could be established. A 60 year old man with no apparent signs of malignancy, (as shown by full-body positron-emission tomography/computed tomography scan) was diagnosed for intramucosal esophageal cancer from his bioptic material. A follow up examination revealed only inflammation thereby hampering actual treatment. Hence in order to move ahead with the actual patient care management conformation regarding authenticity of the belongings of tissue had to be determined. DNA fingerprinting of the initial EGD (esophagogastroduodenoscopy) with tissue obtained from the EUS (endoscopic ultrasound) confirmed same source thereby correctly treating the patient for esophageal carcinoma. Another patient diagnosed prior for the malignancy moved to another clinic for the sound opinion for authenticating the actual treatment because neither EUS nor computed tomography/positron-emission tomography had illustrated the evidences of malignancy. Again in order to address the conflicting nature of the results of different specimens of the same patient, DNA fingerprinting performed on all the previous fixed tissues and a new bioptic tissue, confirmed specimen belongings to the same source (patient) with carcinoma findings (Islam et al. 2013). 
6. Unwanted pregnancies arise as a result of sexual assault or rape cases where the foetus is almost aborted. In three separate cases of this nature, where the seriousness of the crime of rape, incest, or statutory rape had to be determined for the final calculation of paternity includes. (1). Conformation of pregnancy in a woman soldier by an act of rape by a male soldier. DNA profiling of aborted materials from paraffin blocks revealed the suspected male soldier to be a possible father of the foetus where in the paternity index for the suspect was calculated to be 509,768. (2). Authenticity in the allegations of repeated pregnancy by a 14-year-old girl on her stepfather as a result of rape by him. FFPE abortion material of the girl submitted for DNA profiling upon STR analysis revealed the suspect not to be excluded as a possible father of both the fetuses with paternity index of 547,152 side by side exonerating the charges leveled on her boyfriend. (3). Quantum of punishment for alleged rape charges on a father by his 13-year-old daughter that lead into her pregnancy. Repeated samplings of the FFPE gave a mixed foetal, maternal profile as well as a clean foetal profile, thereby authenticating the claim of rape on her father as a possible father of the product of conception, with a paternity index of 10,724 (Reshef et al. 2011).

A little difficulty is experienced in isolating foetal DNA from relatively fresh aborted material or when the appropriate storing conditions of the material are facilitated. Abortion material, as a matter of fact usually consists of the placenta- which in turn is made up of two parts, maternal section and the foetal section. Since both parts of the placenta appear as a single unit, repeated sampling of the material may be important for obtaining a foetal profile. However a collaborative awareness between a forensic man and a pathologist is needed for the preparation and marking of CVS (chorionic villi samples) areas that only originate from the foetus a source that in most cases yield a 'clean' or 'near clean' foetal DNA profile without maternal DNA contamination. Despite the fact of hinderance by the concurrent presence of abundant maternal tissue in chorionic villi of abortion material is, as preferential amplification of excess maternal DNA often prevents detection of fetal genotypes (Reshef et al. 2011).

7. A spindle-shaped blood clot on histological examination of an apparently 21 year old woman with severe physical and mental disabilities revealed a small number of fibrotic chorionic villi surrounded by maternal decidua, blood, and necrosis which immediately raised the suspicion of sexual abuse. In order to identify the father of the product of conception, laser micro-dissected chorionic villi sections separated from archival formalin-fixed paraffin embedded abortion material were subjected to DNA testing. The victim's male relatives (father, brother, paternal grandfather, and maternal grandfather) samples on DNA analysis were excluded due to multiple allelic incompatibilities while her brother whose genotypes were included at each locus suggested the obligate paternal allele of the product of conception with the confirmation of sister-brother incest having a probability of paternity $>99.99999 \%$. However selective cell recovery by way of laser microdisection gravity force recovery (LMDGFR), as evidenced from this research, enabled the amplification of fetal genotypes, which was otherwise masked by the overwhelming presence of maternal DNA in the sample. DNA isolated from nearly 50 microdissected fetal cells possibly generated a reproducible multilocus profile of 15 STR markers for subsequent paternity testing (Robino et al. 2006).

\section{Investigative information for the consideration}

1.1 Alongside the polymorphism virtue use of Short tandem repeat (STR) analysis in forensic identification efforts, it can also be used to detect alterations in diseased tissues. Variations in genetic phenotypes cause allelic changes in the form of expansion/contraction of an allele or simply microsatellite instability (MSI) and loss of heterozygosity ( $\mathrm{LOH}$ - a permanent characteristic feature of cancerous tissues, which make the analysis of cancerous tissues for DNA related forensic identification purposes complicated (DiPerte et al. 2009). So it must be borne in mind that while dealing with DNA extracted from fixed human tissues, a careful caution should always be exercised because of gene alteration in certain diseases which are characterized by frequent genetic and epigenetic aberrations including chromosomal rearrangements, copy number gains losses and DNA methylation particularly what is seen in cancers (Margiotta et al. 2006; Peloso et al. 2003; Pikor et al. 2011; Poetsch et al. 2004; Vauhkonen et al. 2004). The same precautions if not corrected would lead to a wrongful STR typing result interpretation in forensic practices where life or liberty of an individual is always at a stake. As it is noticed that negative consequences on the quality of extracted DNA, inevitably and adversely affects downstream analyses such as PCR or next generation sequencing (NGS) nowadays commonly employed (Lin et al. 2014). Therefore, prior to DNA extraction, it is necessary to incorporate the indispensable help of a 
pathologist who should first be allowed to examine the tissue for any disease presence, the process would thereby help in checking the allelic imbalance pattern on STR profiles.

1.2 In forensic casework there is often an occurrence of body fluid mixtures from different individuals which doesn't deter the value of DNA typing. However standard DNA extraction and STR-typing, at times difficult to employ can be used for the detection of minor component in a mixed stain where its presence is approximately less than one tenth of the major component (Lygo et al. 1994). The same issues can be addressed by way of differential lysis in mixed traces containing semen or Y-STR-typing in male/female mixtures (Gill et al. 1985; Prinz et al. 1997). A recently developed technique of laser microdissection-gravity force recovery (LMDGFR) system enables the analysis of mixed forensic traces, from different contributors by way of morphological discrimination under microscopic controls. For the success of subsequent paternity testing the usage of the technique greatly helped in selective recovery of chorionic villi from archival formalin-fixed, paraffin-embedded abortion material and must always be facilitated.

\section{Assessment of genetic instability in diseased tissues}

Genetic instability either in the form of microsatellite instability (MI) or loss of herozygosity particularly in cancerous samples exercise a great impact on final discrimination results. However, MI can be identified by detection of abnormal alleles at the STR loci compared with the control tissue (healthy). Likewise heterozygote ratio (HR) can be determined by the formula $\mathrm{HR}=\mathrm{A}$ low/A high, where $[\mathrm{A}]$ is the allelic intensity in relative fluorescence units (RFU).

\subsection{STR issue in clinical and forensic research} STR loci for forensic investigations are selected for high rates of heterozygosity, high variability in the population, and a lack of correlation with a physical trait. But in contrast STR regions/loci used in clinical analysis are linked to genes that are highly conserved with an unknown amount of variability. So the accuracy of clinical molecular testing for genetic instability could be compromised by DNA contamination if undetected, leading to erroneous determinations of genetic instability. The same could result in improper diagnosis or therapy to a patient. Reference tissues affected by contamination or diseased tissues by their molecular changes cannot be distinguished from genetic instability. Therefore, it is imperative to individualize all archival samples undergoing genetic testing to ensure for their single source, with no issues of tissue mix-ups. It must always be emphasized that genetic changes in malignant tissue can alter the repeating segments used in the forensic DNA fingerprinting, thus leading to potentially false nonmatches. However, by virtue of mendalian inheritance, each locus is independent from every other locus; the high number of loci used in DNA fingerprinting obviates the same issue.

2.2 Sample mix up issue

The first and the fore most step in suspicion of a mixed profile if any is to evaluate the DNA profile to its entirety. With regard to allelic or genotype assignments at a given locus for the determination of a locus to act as a single source or a mixture, it has been observed that due to some locus specific phenomena like stutter, peak height imbalance, tri-allelic patterns, primer mismatches, and differential amplification, the non-conclusive assignment may thus be permitted. For the determination of a sample being a single source or a mixture (or to conclude the minimum number of contributors), it would be unwise to exclude all other loci and focus only on one locus. It is assumed that a sample originates from more than one individual, when three or more alleles show their presence at one or more loci and or the peak height ratios between a single pair of allelic peaks for one or more loci are below the empirically determined appropriate threshold for heterozygous peak height ratio (Budowle et al. 2009).

\section{Probability calculations}

In absence of actual perpetrator or witness to a crime, DNA evidence for criminal investigations has necessarily to be made in light of uncertainty. However in situations of a DNA profile match between a crime scene sample and the suspect, the terminology changes to probability interpretation rather than certainty. In most forensic situations, a demand for clarification arises for the establishment of exact relationship that exists between two individuals. A probability comparison of evidence under two alternative propositions is measured in the face of likelihood ratio (LR) formalism. The LR approach being the standard universally accepted framework for evaluating the probability of a disputed kinship has been recommended by the Paternity Testing Commission of the International Society of Forensic Genetics (ISFG), for calculating the weight of genetic evidence (Butler 2005; Gjertson et al. 2007; Pinto et al. 2010; Tamura et al. 2015).

\section{Likelihood ratio (LR) approach in paternity testing}

Apart from LR approach, parameters like exclusion probability, probability based on Bayes' theorem etc. are also utilized for parentage testing. The likelihood of one hypothesis is always evaluated relative to another. So in simple terminology LR is the probability of the genetic 
observations assuming the kinship is contrasted with the probability of the same observations under the alternative hypothesis of a different degree of relationship (usually its absence) However, LR approach can also be extended to other relationships as well such as full and half siblings. For mutually independent genotypes, the combined LR is obtained by multiplying the individual LR's according to the product rule. Though biostatistical evaluations of paternity are based on a likelihood ratio principle that yield the Paternity index (PI) and is thus a likelihood ratio obtained on the assumption of probability of types/alleles observed in a hypothesized tested man with the probability of types/alleles observed in a hypothesized random man.

Mathematically PI

Probability of types or observed alleles in a hypothesized Tested man

$=\overline{\text { Probability of types or observed alleles in a hypothesized Random man }}$.

For a value to be inferred as Parentage value, recommendations of the ISFG are followed in typical cut offs of 100 or 1000 .

\section{Conclusions}

In the conclusion it is imperative to address the issues which have a bearing on the routine analysis. With an alarmingly increase in pathological work, there is unfortunately, lack of well-defined standard pre-analytical processes protocols which limit the use of preserved fixed human tissue specimens for DNA analysis particularly when high amounts of DNA are needed. However, few aspects warrant special care before a DNA profile is examined for final interpretation. With an ever increasing importance of DNA in the identification process, it is desirable that forensic research work should also focus on clinical tissue samples (fixed specimens) as a source for DNA typing. So, that a greater role is played in paternity testing cases and unknown bodies identification cases. It would also aid in resolving issues stemming from pathology mix-ups and contaminations. A caution should be exercised regarding genetic changes in malignant tissue that can alter the repeating segments used in the forensic DNA fingerprinting, thus leading to potentially false nonmatches. However, by virtue of Mendalian inheritance, each locus is independent of other locus; the high number of loci used in DNA fingerprinting obviates the same issue because the certainty of identity with one locus is less than 0.01 , and is $1.0 \times 10^{-15}$ for 12 or more loci making STR a very effective method for the identification. The same objective may be achieved if DNA extraction techniques are optimized overcoming the common obstacles inherent to the sample, that include limited/low quantities of DNA, old and degraded DNA and substrate inhibitors usually collected with the fixed sample. The optimization at least will allow the recovery of good quality DNA without introducing any chemicals or reagents that may have deleterious effect on the downstream analytical procedures, where more loci are amplified for better discrimination.

Another noteworthy point that warrants special consideration is an alarmingly increase in the number of rape cases in the country, where in majority of cases, paternity has to be ascertained for fair judgment. It is thus essential that forensic labs should be equipped with experts specialized in histological procedures to carry out the separation procedure in order to mark CVS (chorionic villi sample) areas so that uncontaminated foetal profile is obtained.

Simultaneously with an alarmingly increase in pathological work, and a lack of well-defined standard pre-analytical processes protocols, the issues of tissue contamination, sample mix up/interchanging, absence of correct tissue part, and the presence of extraneous tissue in paraffin blocks have got well recognition in pathology departments. The conflicting results so observed on follow-ups, is a matter of great concern for both the physician and the patient in their forward management. Unfortunately the patient at times has to bear the whole brunt for the consequences appearing to be dramatic. It is required that while working with preserved formalin fixed and formalin fixed, paraffin embedded tumor tissue specimens, a careful interpretation of microsatellite instability (MSI) and loss of heterozygosity (LOH) should be thoroughly made. The best strategy is to take the help of a pathologist who by his/her knowledge makes a partition/ separation between cancerous tissues and adjacent normal tissues from each other after microscopic evaluation with H.E. staining. After the procedure, DNA extraction should be followed so that better discrimination is obtained and proper reporting could be made in criminal justice system.

\section{Acknowledgements \\ The author is thankful to UGC New Delhi for the financial support provided in the form of BSR fellowship to carry out the research. Sincere gratitude is duly expressed to Dr. Sahar Ibrahim, Lecturer Faculty of Medicine Alexandria University, Egypt for reading and editing this manuscript despite her busy schedule. Special thanks to Prof. Sabhiya Majid and Prof. Ruby Reshi, Heads Department of Biochemistry and Pathology, Govt Medical College Srinagar respectively for their constant support, encouragement and providing lab facilities along with easy access to fixed samples.}

\section{Funding}

University Grants Commission New Delhi India. No: 8957; Dated: 06/08/2013.

\section{Availability of data and materials}

Data sharing is not applicable to this article as no datasets were generated or analyzed during the current study.

\section{Authors' contributions}

This study was conceived and designed by SU and RKG. SU and FN acquired the data. Analysis and interpretation of data was completed by SU, FN and RKG. SU drafted the manuscript. RKG provided critical revision. All authors read and approved the final manuscript.

Ethics approval and consent to participate

Institutional Ethics Committee (IEC) Punjabi University, Patiala. No: 33; Dated: 21/01/2016. 


\section{Consent for publication}

Not applicable.

\section{Competing interests}

The authors declare that they have no competing interests.

\section{Publisher's Note}

Springer Nature remains neutral with regard to jurisdictional claims in published maps and institutional affiliations.

\section{Author details}

${ }^{1}$ Department of Forensic Science, Punjabi University, Patiala, Punjab 147002, India. ${ }^{2}$ Department of Forensic Medicine \& Toxicology, Government Medical College, Srinagar, Kashmir (J\&K) 190010, India

Received: 3 May 2017 Accepted: 22 November 2017

Published online: 06 December 2017

\section{References}

Banaschak S, Chesne AD, Brinkmann B (2000) Multiple interchanging of tissue samples in cases of breast cancer. Forensic Sci Int 113(1-3):3-7

Blow N (2007) Tissue preparation: tissue issues. Nature 448:959-963

Budimlija Z, Lu C, DiPerte GA, Seifarth J, Popiolek D, Fogt F, Prinz M (2009) Malignant tumors and forensics - dilemmas and proposals. Croat Med J 50:218-227

Budowle B, Onorato AJ, Callaghan TF, Della Manna A, Gross AM, Guerrieri RA, Luttman JC, McClure DL (2009) Mixture interpretation: defining the relevant features for guidelines for the assessment of mixed DNA profiles in forensic casework. J Forensic Sci 54(4):810-821

Butler JM. (2005) Forensic DNA typing: biology, technology, and genetics of STR markers, 2nd ed. Elsevier Academic Press, New York

Chugh SS, Senashova O, Watts A, Tran PT, Zhou Z, Gong Q, Titus JL, Hayflick SJ (2004) Postmortem molecular screening in unexplained sudden death. J Am Coll Cordiol 43:1625-1629

DiPerte GLA, Wurmbach E, Budimlija ZM, Jian B, Fogt F, Prinz M (2009) Forensic STR analysis reveals DNA contamination previously undetected during clinical analysis of chronically inflamed tissues. Forensic Sci Int Genet 2:14-16

Doolan A, Langlois N, Chiu C, Ingles J, Lind JM, Semsarian C (2008) Postmortem molecular analysis of KCNQ1 and SCN5A genes in sudden unexplained death in young Australians. Int J Cardiol 127:138-111

Farrugia A, Keyser C, Ludes B (2010) Efficiency evaluation of a DNA extraction and purification protocol on archival formalin-fixed and paraffin-embedded tissue. Forensic Sci Int 194(1-3):e25-e28

Gilbert MTP, Haselkorn T, Bunce M, Sanchez JJ, Lucas SB, Jewell LD, Marck EV, Worobey M (2007) The isolation of nucleic acids from fixed, paraffin-embedded tissues-which methods are useful when? PLoS One 2(6):e537

Gill P, Jeffreys AJ, Werrett DJ (1985) Forensic application of DNA fingerprints. Nature 318:577-579

Gillio-Tos A, Demarco L, Fiano V, Bragado FG, Dikshit R, Boffetta P, Merletti F (2007) Efficient DNA extraction from 25-year-old paraffin-embedded tissues: study of 365 samples. Pathology 39(3):345-348

Gjertson DW, Brenner CH, Baur MP, Carracedo A, Guidet F, Luque JA, Lessig R, Mayr WR, Pascali VL, Prinz M, Schneider PM, Morling N (2007) ISFG: recommendations on biostatistics in paternity testing. Forensic Sci Int Genet $1: 223-231$

Huijsmans CJJ, Damen J, Linden JCV, Savelkoul PHM, Hermans MHA (2010) Comparative analysis of four methods to extract DNA from paraffin embedded tissues: effects on downstream molecular applications. BMC Res Notes 3:239

Islam S, Miller ED, Patel N, DePetris G, Highsmith EW, Fleischer DE (2013) The use of DNA fingerprinting to resolve conflicting results in patients with suspected gastrointestinal malignancy. J Clin Gastroenterol 47:239-241

Lin L, Vega M, Jones RT, Ziaugra L, Dolcen DN, Sunkavalli A, Schubert L, Lepine JR, Thorner AR, Ducar MD, Hahn WC, Meyerson ML, MacConaill LE, Hummelen PV (2014) Improved FFPE DNA extraction for next generation sequencing using adaptive focused acoustics technology. Cancer Res 74:abstract 4286

Liu Y, Li L, Li C, Zha Z (2009) Allelic alterations of STR's in archival paraffin embedded tissue as DNA source for paternity testing. Forensic Sci Int Genet 2(1):12-13

Lygo JE, Johnson PE, Holdaway DJ, Woodroffe S, Whitaker JP, Clayton TM (1994) The validation of short tandem repeat (STR) loci for use in forensic casework Int J Legal Med 107:77-89
Margiotta G, Coletti A, Lancia M, Lottanti L, Carnevali E, Bacci M (2006) Evaluation of allelic alterations in STR in different kinds of tumors and formalin fixed tissues-possible pitfalls in forensic casework. Int Congr Ser 1288:519-521

Peloso G, Grignani P, Rosso R, Previdere C (2003) Forensic evaluation of tetranucleotide STR instability in lung cancer. Int Congr Ser 1239:719-721

Pikor LA, Enfield KSS, Cameron H, Lam WL (2011) DNA extraction from paraffin embedded material for genetic and epigenetic analyses. J Vis Exp 49. 10. 3791/2763 http://www.jove.com/details.php?id=2763

Pinto N, Leonor Gusmao L, Antonio Amorim A (2010) Likelihood ratios in kinship analysis: contrasting kinship classes, not genealogies. Forensic Sci Int Genet 4(3):218-219

Poetsch M, Petersmann A, Woenckhaus C, Protzel C, Dittberner T, Lignitz E, Kleist B (2004) Evaluation of allelic alterations in short tandem repeats in different kinds of solid tumors-possible pitfalls in forensic casework. Forensic Sci Int 145:1-6

Popiolek DA, Prinz MK, West AB, Nazzaruolo BL, Estacio SM, Budimlija ZM (2003) Multiplex DNA short tandem repeat analysis. A useful method for determining the provenance of minute fragments of formalin-fixed, paraffin-embedded tissue. Am J Clin Pathol 120(5):746-751

Prinz M, Boll K, Baum H, Shaler B (1997) Multiplexing of Y chromosome specific STRs and performance for mixed samples. Forensic Sci Int 85:209-218

Raina A, Yadav B, Ali S, Dogra TS (2011) Identification of tumor specimens by DNA analysis in a case of histocytological paraffin tissue block swapping. Croat Med J 52:410-414

Reshef A, Barash M, Voskoboinik L, Brauner P, Gafny R (2011) STR typing of formalin-fixed paraffin embedded (FFPE) aborted foetal tissue in criminal paternity cases. Sci Justice 51:19-23

Robino C, Barilaro MR, Gino S, Chiarle R, Palestro G, Torre C (2006) Incestuous paternity detected by STR typing of chorionic villi isolated from archival formalin fixed paraffin embedded abortion material using laser microdissection. J Forensic Sci 51(1):90-92

Romero R, Juston AC, Ballantyne J, Henry BE (1997) The applicability of formalin-fixed and formalin-fixed paraffin embedded tissues in forensic DNA analysis. J Forensic Sci 42:708-714

Tamura T, Osawa M, Ochiai E, Suzuki T, Nakamura T (2015) Evaluation of advanced multiplex short tandem repeat systems in pairwise kinship analysis. Legal Med 17(5):320-325

Vauhkonen $\mathrm{H}$, Hedman M, Vauhkonen M, Kataja M, Sipponen P, Sajantila A (2004) Evaluation of gastrointestinal cancer tissues as a source of genetic information for forensic investigations by using STRs. Forensic Sci Int 139(2):159-167

\section{Submit your manuscript to a SpringerOpen ${ }^{\circ}$ journal and benefit from:}

- Convenient online submission

- Rigorous peer review

- Open access: articles freely available online

- High visibility within the field

Retaining the copyright to your article

Submit your next manuscript at $>$ springeropen.com 